\title{
Thermodynamic Properties of Real Porous Combustion Reactor under Diesel Engine-Like Conditions
}

\author{
M. Weclas, ${ }^{1}$ J. Cypris, ${ }^{1}$ and T. M. A. Maksoud ${ }^{2}$ \\ ${ }^{1}$ Institute of Vehicle Technology (IFZN), Faculty of Mechanical Engineering, Georg Simon Ohm University of Applied Sciences, \\ Nuremberg, Kesslerplatz 12, 90489 Nuremberg, Germany \\ ${ }^{2}$ Faculty of Advanced Technology, University of Glamorgan, Pontypridd CF37 1DL, UK
}

Correspondence should be addressed to M. Weclas, miroslaw.weclas@ohm-hochschule.de

Received 7 June 2011; Revised 2 November 2011; Accepted 3 November 2011

Academic Editor: L. De Goey

Copyright (c) 2012 M. Weclas et al. This is an open access article distributed under the Creative Commons Attribution License, which permits unrestricted use, distribution, and reproduction in any medium, provided the original work is properly cited.

\begin{abstract}
Thermodynamic conditions of the heat release process under Diesel engine-like conditions in a real porous combustion reactor simulated in a special combustion chamber were analyzed. The same analyses were performed for a free volume combustion chamber, that is, no porous reactor is applied. A common rail Diesel injection system was used for simulation of real engine fuel injection process and mixture formation conditions. The results show that thermodynamic of the heat release process depends on reactor heat capacity, pore density, specific surface area, and pore structure, that is, on heat accumulation in solid phase of porous reactor. In real reactor, the gas temperature and porous reactor temperature are not equal influenced by initial pressure and temperature and by reactor parameters. It was found that the temperature of gas trapped in porous reactor volume during the heat release process is less dependent on air-to-fuel-ratio than that observed for free volume combustion chamber, while the maximum combustion temperature in porous reactor is significantly low. As found this temperature depends on reactor heat capacity, mixture formation conditions and on initial pressure. Qualitative behavior of heat release process in porous reactors and in free volume combustion chamber is similar, also the time scale of the process.
\end{abstract}

\section{Introduction}

One of novel combustion technologies is combustion in porous reactors $(\mathrm{PM})$. It is possible to perform a homogeneous and flameless heat release process inside a stationary operating porous reactor resulting in a nearly zero-emission level [1-6]. The combustion process is characterized by a high power density in large dynamic range under stable combustion conditions. Such features of combustion process would be much advantaged in application to internal combustion engine. However, the process conditions in engine are much more complex: the process is nonstationary and is performed under high pressure with requirement of mixture formation directly inside the combustion reactor. Durst and Weclas [7-9] proposed engine concept entails mixture formation and combustion in porous reactor characterized by a nearly zero-emission level and indicating potential for increasing cycle efficiency. Furthermore, the effect of reactor heat capacity resulting in lowering of combustion temperature as well as internal heat recuperation during the engine cycle changes the thermodynamic conditions of the process as compared to conventional system, for example, Otto and Diesel cycles [8-13]. In a real engine, the fuel must directly be injected into combustion reactor and must be distributed throughout the reactor volume together with vaporization and mixing with combustion air. The hot reactor is used as a three-dimensional "hot-spot" igniter, and the heat release process should be performed in the reactor volume only. This requires that the fuel should be injected in a short period of time close to the TDC of compression stroke. Such complex process conditions make on the one hand description of the process itself and on the other hand a practical realization of engine with combustion in porous reactor very difficult. The reactor temperature and its distribution in porous medium volume (temperature of solid phase of the reactor and temperature of the gas trapped inside reactor volume) together with mixture formation inside the reactor define the conditions for thermal ignition 


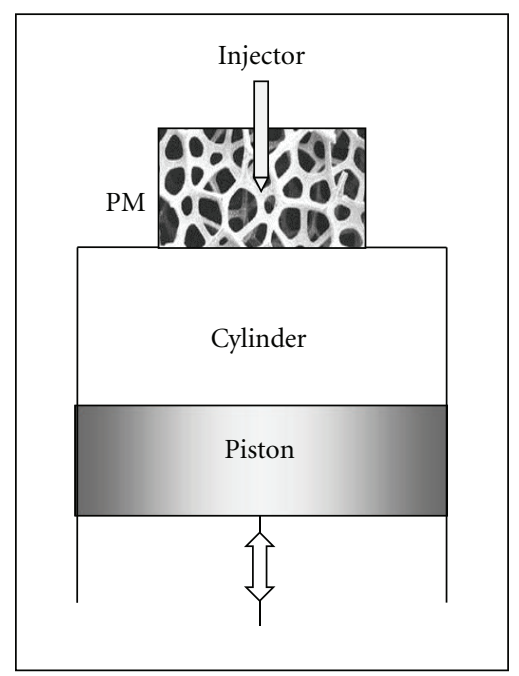

(a)

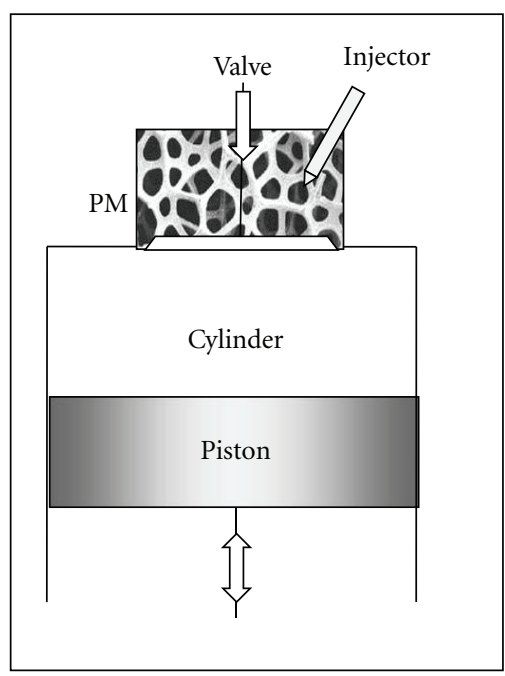

(b)

Figure 1: Principle of internal combustion engine with mixture formation and combustion in porous reactor (as proposed by Durst and Weclas [8]): (a) reactor with permanent contact with cylinder content; (b) reactor with periodic contact with cylinder content.

and following heat release process. This is because the reactor heat capacity and large specific surface area for interphase heat transfer inside the reactor volume change the thermodynamic conditions of the process $[14,15]$. Numerical simulations of heat release process in porous reactors under engine-like conditions usually use simplified conditions and cannot properly describe conditions found in a real combustion reactor $[6,10,12,13]$. The present work describes thermodynamic of real porous reactor under Diesel enginelike combustion conditions. The fuel is directly injected into a porous reactor resulting in real engine mixture formation conditions. The effect of reactor heat capacity, pore density, and pore structure is also considered in this investigation. The authors concentrate on describing the thermodynamic conditions of the porous reactor with a heat release process under Diesel engine-like conditions. This is compared to a free volume Diesel combustion. The focus of this work is to analyse reactor thermodynamics, but the detailed analysis of the heat release process itself is outside the scope of this work (more information is available in [14-16]).

An engine concept with mixture formation and combustion in a porous reactor is described in Section 2.1. Definition and description of considered thermodynamic systems are given in Section 2.2, where a free volume system is compared to the ideal and real porous reactors. An engine simulator (special combustion chamber) used in present investigation for simulation of real reactor operation under Diesel engine-like conditions is also presented in this section. The next, Section 2.3, presents description of thermodynamic conditions of all considered systems. In Section 2.4, the authors present simplified analysis of heat recuperated (accumulated) in the reactor solid phase as compared to free volume system. Results of experimental investigation for a real combustion reactor in comparison to free volume Diesel engine conditions are presented and discussed in Section 3. Section 4 summarizes presented results.

\section{Engine Concept with Heat Release in Porous Reactor and Corresponding Thermodynamic Systems Describing Engine Conditions}

\subsection{Short Description of Engine Concept with Combustion} in Porous Reactor. Durst and Weclas [7-9] proposed engine concept with mixture formation and combustion processes in porous reactor. Application of a combustion porous reactor to engine allows realization of homogeneous and flameless combustion process characterized by a nearzero-emission level. Heat recuperation in porous reactor may allow increase of engine-cycle efficiency resulting in reduction of $\mathrm{CO}_{2}$ emissions. Heat accumulation in porous reactor results in significantly lowered combustion temperature permitting nearly zero $\mathrm{NO}_{x}$ level. This is the most important difference between the combustion processes in conventional Diesel engine and in engine with combustion in porous reactor. The latter kind of engine may be realized in two different ways [8]: engine in which the PM reactor has permanent contact with the cylinder content (open chamber Figure 1(a)) and engine in which the PM reactor has periodic contact with the cylinder content (closed chamber Figure 1(b)). In the case of a closed chamber, it is assumed that the valve in the PM chamber opens near to the TDC of compression and remains open during the expansion stroke in the cylinder. In the open-chamber system, as considered in the present work, the porous reactor has permanent contact with the cylinder content (gas) and the most important are processes occurring close to the TDC of compression in a constant volume of porous reactor. These processes are fuel injection into reactor, fuel distribution throughout reactor volume, fuel vaporization and mixing with air, as well as thermal ignition and heat release. First of all, the fuel must directly be injected into porous reactor and distributed throughout reactor volume. The authors performed extensive investigations on 


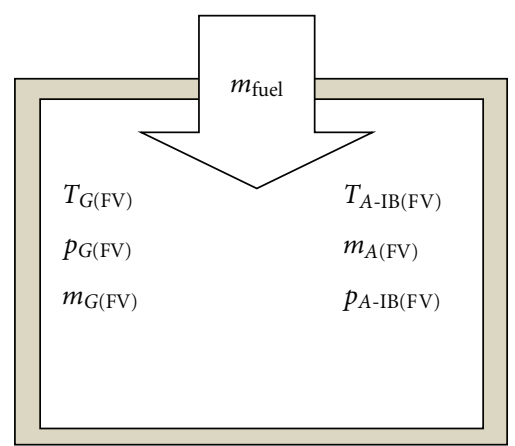

(a)

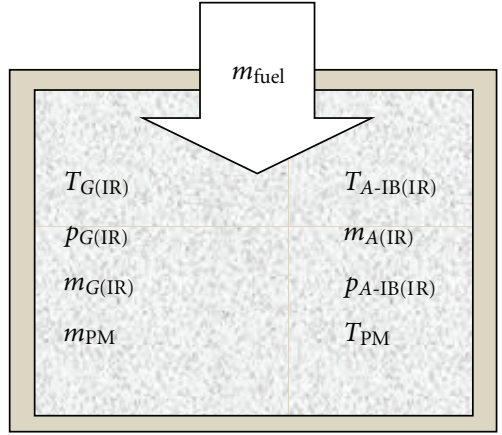

(b)

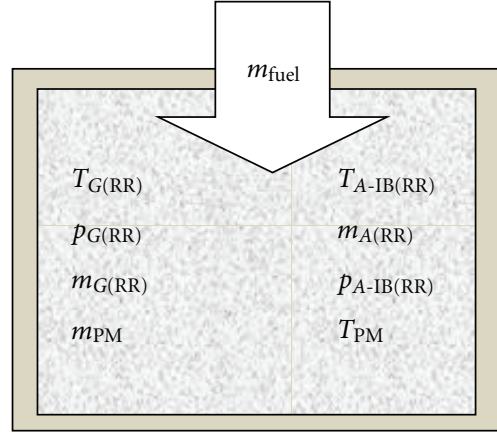

(c)

Figure 2: Model of considered systems without heat losses through the system walls (description in text): (a) free volume system, (b) ideal porous reactor, and (c) real porous reactor.

Table 1

\begin{tabular}{|c|c|c|c|}
\hline \multirow{2}{*}{ Parameter } & \multicolumn{3}{|c|}{ System/reactor } \\
\hline & FV & IR & $\mathrm{RR}$ \\
\hline Mass of air supplied to the system before fuel injection starts & $m_{\mathrm{A}(\mathrm{FV})}$ & $m_{\mathrm{A}(\mathrm{IR})}$ & $m_{\mathrm{A}(\mathrm{RR})}$ \\
\hline Mass of gas trapped in the system after fuel injection & $m_{\mathrm{G}(\mathrm{FV})}$ & $m_{\mathrm{G}(\mathrm{IR})}$ & $m_{\mathrm{G}(\mathrm{RR})}$ \\
\hline Mass of porous reactor & - & $m_{\mathrm{PM}}$ & $m_{\mathrm{PM}}$ \\
\hline Temperature of porous reactor at the moment of fuel injection & - & $T_{\mathrm{PM}-\mathrm{IB}}$ & $T_{\mathrm{PM}-\mathrm{IB}}$ \\
\hline Temperature of porous reactor after fuel injection & - & $T_{\mathrm{PM}}$ & $T_{\mathrm{PM}}$ \\
\hline Air temperature at the moment of fuel injection & $T_{\mathrm{A}-\mathrm{IB}(\mathrm{FV})}$ & $T_{\mathrm{A}-\mathrm{IB}(\mathrm{IR})}$ & $T_{\mathrm{A}-\mathrm{IB}(\mathrm{RR})}$ \\
\hline Air pressure at the moment of fuel injection & $p_{\mathrm{A}-\mathrm{IB}(\mathrm{FV})}$ & $p_{\mathrm{A}-\mathrm{IB}(\mathrm{IR})}$ & $p_{\mathrm{A}-\mathrm{IB}(\mathrm{RR})}$ \\
\hline Gas temperature after fuel injection & $T_{\mathrm{G}(\mathrm{FV})}$ & $T_{\mathrm{G}(\mathrm{IR})}$ & $T_{\mathrm{G}(\mathrm{RR})}$ \\
\hline Gas pressure after fuel injection & $p_{\mathrm{G}(\mathrm{FV})}$ & $p_{\mathrm{G}(\mathrm{IR})}$ & $p_{\mathrm{G}(\mathrm{RR})}$ \\
\hline
\end{tabular}

the Diesel jet/spray interaction with porous structure and described a multijet splitting as a basic process responsible for three-dimensional spray spreading [17-22]. Owing to the complexity of this process and to the role of reactor structure, pore size and pore geometry in interaction of Diesel spray with porous-structure, extended experimental investigations are still required for better understanding of the process. This extension concerns direct measurements of spray distribution inside porous reactor volume under high reactor temperatures (reactors are optically not transparent).

2.2. Definition of Considered Thermodynamic Systems. There are three system considered in this analysis, Figure 2 and Table 1.

(i) Free volume system (Figure 2(a)): at the moment of fuel injection, the system consists of mass of air trapped in the combustion chamber volume.

(ii) Ideal porous reactor (Figure 2(b)): at the moment of fuel injection, the system consists of mass of air trapped in the porous reactor volume having mass $m_{\mathrm{PM}}$; at any instant of time, the gas temperature is equal to porous reactor temperature $T_{\mathrm{G}(\mathrm{IR})}=T_{\mathrm{PM}}$.

(iii) Real porous reactor (Figure 2(c)): at the moment of fuel injection, the system consists of mass of air trapped in the porous reactor volume having mass
$m_{\mathrm{PM}}$; at any instant of time after fuel injection starts, the gas temperature is not equal to porous reactor temperature $T_{\mathrm{G}(\mathrm{RR})} \neq T_{\mathrm{PM}}$.

Both porous reactors are made of a highly porous structure having porosity higher than $80 \%$ (in real case more than $90 \%)$. In all considered cases, the fuel is directly supplied into the combustion chamber/reactor using a common-rail Diesel injection system with electronically controlled injector. The mass of injected fuel results from the combination of injection pressure and injection duration. For both models with PM, it is assumed that the mass of reactor is much higher than the mass of gas trapped in the reactor volume

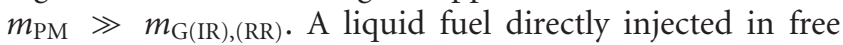
volume combustion system corresponds to the mixture formation conditions typical for Diesel engine conditions (no air motion is considered). Direct fuel injection into porous reactor changes fuel distribution in space [20-22] resulting in different mixture formation conditions. This process, however, is significantly dependent on pore density, pore size, pore structure, as well as injection conditions (especially injection pressure).

In the present investigation, a real engine with mixture formation and combustion in porous reactor, as proposed by Durst and Weclas [8], is considered and simulated in a special combustion chamber as shown in Figure 3. A principle of internal combustion engine with mixture formation and 


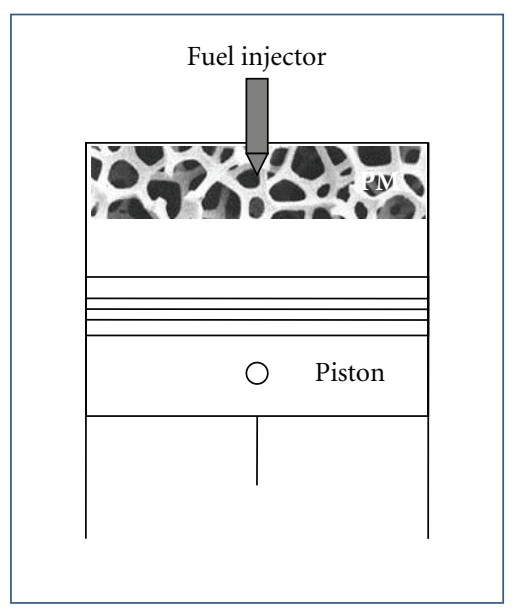

(a)

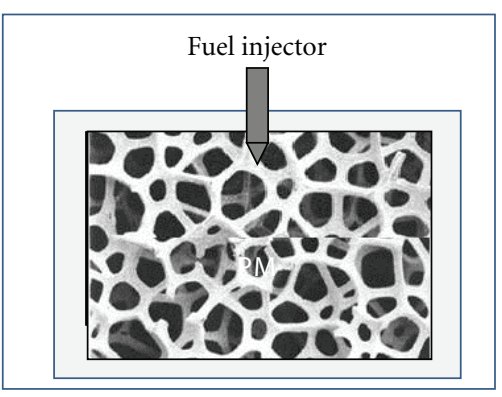

(b)

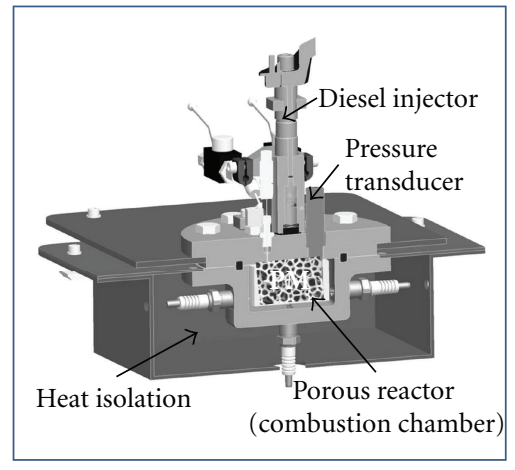

(c)

FIGURE 3: Modeling of real engine realizing combustion in porous reactor (a) with a constant volume reactor (b) and engine simulator used in preset investigation (c).

combustion in porous reactor assumes that, at the TDC of compression, the only available volume in the cylinder is the volume of porous reactor (Figure 3(a)). Fuel is directly injected into porous reactor and is completely trapped in its volume. For investigating the mixture formation and heat release process in engine, it is possible to reduce the system to the reactor volume corresponding to the engine conditions at TDC of compression (Figure 3(b)). For experimental investigation, these conditions have been realized in a special engine simulator built in the form of an isolated constant volume high-temperature and highpressure combustion chamber equipped with common rail Diesel injection system and electronically controlled fuel injector. The fuel is then directly injected into porous reactor volume [16]. The high-pressure combustion chamber with porous reactor is mounted inside a low-pressure chamber to act as heat isolation (Figure 3(c)). Reactor can be heated electrically, and the current is supplied using electrodes of spark plugs mounted in the chamber. The combustion (dry) air is supplied to the chamber under selected pressure, and the flow rate control valve permits supply of required mass of air (for control of air excess ratio $\lambda$ ). Pressure transducer mounted in the combustion chamber allows very precise and highly time-resolved measurements of pressure changes after fuel injection starts. This pressure history allows the reconstruction of heat release process (combustion) and corresponding temperatures. It is assumed (and experimentally verified) that, during the heat release process, the heat losses through the combustion chamber walls are negligible.

\subsection{Thermodynamics of Free Volume System and Porous} Reactor. The mass of fuel $m_{\text {fuel }}$ injected into system without heat losses through the system walls (see Figure 2) represents amount of energy supplied to the system $E_{\text {in }}$ at initial thermodynamic conditions at the time instance of injection starts $T_{\mathrm{IB}}$ and $p_{\mathrm{IB}}$ :

$$
E_{\text {in }}=m_{\text {fuel }} \cdot H_{u} \text {, }
$$

where $H_{u}$ is the heating value of injected fuel. During combustion process, this energy is converted into heat $Q_{\text {in }}$ :

$$
Q_{\text {in }}=\eta \cdot m_{\text {fuel }} \cdot H_{u} \text {, }
$$

where $\eta$ is the energy conversion efficiency. For the reason of the following analysis, it is assumed that, for the same amount of injected fuel, the conversion efficiency in free volume system $\eta_{\mathrm{FV}}$ and real porous reactor $\eta_{\mathrm{RR}}$ is the same.

In the case of free volume system with no heat losses through the system walls (Figure 2(a)) heat supplied to the system results in increasing internal energy $\Delta U_{\mathrm{G}(\mathrm{FV})}$ of the gas trapped in this volume:

$$
Q_{\text {in }}=\Delta U_{\mathrm{G}(\mathrm{FV})} .
$$

Increase of internal energy corresponds to the change in the gas temperature $\Delta T_{\mathrm{G}(\mathrm{FV})}$ and gas pressure $\Delta p_{\mathrm{G}(\mathrm{FV})}$ after injection starts (for initial air conditions $T_{\mathrm{A}-\mathrm{IB}(\mathrm{FV})}$ and $\left.p_{\mathrm{A}-\mathrm{IB}(\mathrm{FV})}\right)$ :

$$
\begin{gathered}
\Delta T_{\mathrm{G}(\mathrm{FV})}=T_{\mathrm{G}(\mathrm{FV})}-T_{\mathrm{A}-\mathrm{IB}(\mathrm{FV})}, \\
\Delta p_{\mathrm{G}(\mathrm{FV})}=p_{\mathrm{G}(\mathrm{FV})}-p_{\mathrm{A}-\mathrm{IB}(\mathrm{FV})} .
\end{gathered}
$$

This pressure which is directly measured in an engine simulator (see Figure 3 ) is dependent on direct heat release rate.

In the case of system with porous reactor, the increase of internal energy as a result of heat supplied to the system $Q_{\text {in }}$ is distributed in two components:

$$
Q_{\text {in }}=\Delta U_{\mathrm{G}(\mathrm{PM})}+\Delta U_{\mathrm{PM}} \text {, }
$$

where $\Delta U_{\mathrm{G}(\mathrm{PM})}$ is the change in internal energy of the gas trapped in the porous reactor after fuel injection starts and $\Delta U_{\mathrm{PM}}$ is the internal energy change of porous reactor. Internal energy change of the gas results in change of the gas temperature $\Delta T_{\mathrm{G}(\mathrm{PM})}$ trapped in the reactor volume, and correspondingly the change in internal energy 
of the porous reactor results in $\Delta T_{\mathrm{PM}}$. In the case of ideal reactor (Figure 2(b)), it is assumed that the gas and reactor temperatures are the same at any instant of time after energy supply. From this assumption follows

$$
\Delta T_{\mathrm{G}(\mathrm{IR})} \simeq \Delta T_{\mathrm{PM}} .
$$

Under consideration of a high heat capacity reactor, which is many times higher than heat capacity of the gas trapped in the reactor volume (by a factor of $10^{3}$ and more) the reactor temperature can only slightly change during the heat release process. This means that the gas temperature in an ideal reactor changes also very slightly. For almost constant gas temperature, the pressure changes in the ideal reactor volume resulting from the heat release process are very small $\Delta p_{\mathrm{G}(\mathrm{IR})} \sim 0$.

For real reactor conditions (see Figure 2(c)), the temperature of gas trapped in the reactor volume is not immediately equal the reactor temperature $\left(\Delta T_{\mathrm{G}(\mathrm{RR})} \neq \Delta T_{\mathrm{PM}}\right)$. Thus, the change in internal energy of the system can be expressed as:

$$
\begin{gathered}
Q_{\text {in }}=m_{\mathrm{G}(\mathrm{RR})} \cdot c_{\mathrm{VG}} \cdot \Delta T_{\mathrm{G}(\mathrm{RR})}+m_{\mathrm{PM}} \cdot c_{\mathrm{VPM}} \cdot \Delta T_{\mathrm{PM}}, \\
\Delta T_{\mathrm{G}(\mathrm{RR})}=\frac{Q_{\mathrm{in}}-\left(m_{\mathrm{PM}} \cdot c_{\mathrm{VPM}} \cdot \Delta T_{\mathrm{PM}}\right)}{m_{\mathrm{G}(\mathrm{RR})} \cdot c_{V G}} \longrightarrow \Delta p_{\mathrm{G}(\mathrm{RR})} .
\end{gathered}
$$

Please note that the heat capacities $c_{\mathrm{VG}}$ and $c_{\mathrm{VPM}}$ are considered as mean values of those at initial and final temperatures.

According to the assumptions made in Figure 2 and to above analysis, it is possible to express the following relations:

$$
\begin{gathered}
\Delta T_{\mathrm{G}(\mathrm{FV})}>\Delta T_{\mathrm{G}(\mathrm{RR})} \gg \Delta T_{\mathrm{G}(\mathrm{IR})}, \\
\Delta p_{\mathrm{G}(\mathrm{FV})}>\Delta p_{\mathrm{G}(\mathrm{RR})} \gg \Delta p_{\mathrm{G}(\mathrm{IR})} .
\end{gathered}
$$

For a real reactor, it is possible also to express that

$$
\Delta T_{\mathrm{G}(\mathrm{RR})}=\Delta T_{\mathrm{G}(\mathrm{FV})}-\left(K \cdot \Delta T_{\mathrm{PM}}\right),
$$

where $K$ is the factor characterizing heat capacity of the reactor and heat transfer between gas and reactor solid phase.

In all considered models (FV, IR, and RR), it was assumed that the fuel is homogeneously distributed throughout the combustion chamber volume. This is the reason why the assumption of equal energy conversion efficiency in FV and RR systems was made. In a real case, however, fuel injection conditions in a free volume and in porous reactor are different, especially regarding fuel distribution in space, vaporization, and mixing with air [16, 20-22]. Complete and quantitative description of real engine conditions is at the present time too complex; however, these differences are considered in analyses presented below.

2.4. Heat Accumulation in Porous Reactor. The following analysis gives insight into amount of heat transferred to the gas and to porous reactor based on the maximum temperature after energy supply (fuel injection starts). Real reactor conditions are considered with a simplification assumption that the energy conversion rate into heat is constant and is the same for free volume system and for porous combustion reactor. For a free volume system, the increase of internal energy based on the maximum combustion temperature is expressed as:

$$
\Delta U_{\mathrm{G}(\mathrm{FV})}=U_{\mathrm{G}\left(\mathrm{T}_{\max }\right)}-U_{\mathrm{G}\left(\mathrm{T}_{\mathrm{IB}}\right)},
$$

where $U_{\mathrm{G}\left(\mathrm{T}_{\max }\right)}=m_{\mathrm{G}(\mathrm{FV})} \cdot c_{\mathrm{VG}} \cdot T_{\mathrm{G}(\mathrm{FV})}$ and $U_{\mathrm{G}\left(\mathrm{T}_{\mathrm{IB}}\right)}=m_{\mathrm{A}(\mathrm{FV})}$. $c_{\mathrm{VA}} \cdot T_{\mathrm{A}-\mathrm{IB}(\mathrm{FV})}$.

The authors have defined the efficiency $\eta$ of the total supplied energy conversion into heat based on the maximum temperature of the heat release process, that is, until the time instance of achieving this maximum temperature:

$$
\begin{gathered}
\eta_{\mathrm{FV}}=\frac{\Delta U_{\mathrm{G}(\mathrm{FV})}}{E_{\mathrm{in}}}, \\
\eta_{\mathrm{FV}}=\frac{m_{\mathrm{G}(\mathrm{FV})} \cdot c_{\mathrm{VG}} \cdot T_{\mathrm{G}(\mathrm{FV})}-m_{\mathrm{A}(\mathrm{FV})} \cdot c_{\mathrm{VA}} \cdot T_{\mathrm{A}-\mathrm{IB}(\mathrm{FV})}}{m_{\text {fuel }} \cdot H_{u}} \cdot 100 \%,
\end{gathered}
$$

where $m_{G}=m_{\text {fuel }}(1+14.5 \cdot \lambda)$.

It must be noted that, in both free volume and in porous reactor systems, the fuel is directly injected into the system and the resulting mixture is not homogeneous in space. The time taken to achieve maximum temperature is not necessarily the same time where all the energy supplied is turned into heat. In porous reactor (depending on the injection parameters), this homogeneity can be improved. In the case of porous reactor, the system consists of gas trapped in porous reactor having much higher heat capacity as the gas has. In this case, the amount of heat transferred to the reactor solid phase based on the comparison to total amount of heat supplied under free volume conditions can be expressed as (based on $T_{\max }$ )

$$
\eta_{\mathrm{RR}(\mathrm{PM})}=\frac{\Delta U_{\mathrm{RR}(\mathrm{PM})}}{\mathrm{Q}_{\mathrm{in}}} \cdot 100 \%
$$

under simplified assumption that $Q_{\text {in }(\mathrm{PM})}=Q_{\text {in }(\mathrm{FV})}$; thus,

$$
\eta_{\mathrm{RR}(\mathrm{PM})}=\frac{\Delta U_{\mathrm{G}(\mathrm{FV})}-\Delta U_{\mathrm{G}(\mathrm{PM})}}{\Delta U_{\mathrm{G}(\mathrm{FV})}} \cdot 100 \% .
$$

\section{Heat Release in a Real Porous Reactor under Diesel Engine-Like Conditions}

Examples of pressure history and corresponding temperature for free volume system (Diesel engine-like conditions) are shown in Figure 4. Data are plotted for initial gas temperature $T_{\mathrm{A}-\mathrm{IB}(\mathrm{FV})}=500^{\circ} \mathrm{C}$ at different initial pressures $p_{\mathrm{A}-\mathrm{IB}(\mathrm{FV})}$. The mass of injected fuel is $23.8 \mathrm{mg}$. The pressure history shows a strong dependence of the heat release process on the initial pressure. Generally, the higher the initial chamber pressure is the faster is the heat release process and the shorter ignition delay period $[16,23]$. Also the corresponding pressure peak level increases with initial pressure. This is not the case for combustion temperature which does not up with increasing pressure. This is due to the fact that with increasing initial pressure, air excess ratio also increases, which controls the combustion temperature. Similar analysis 


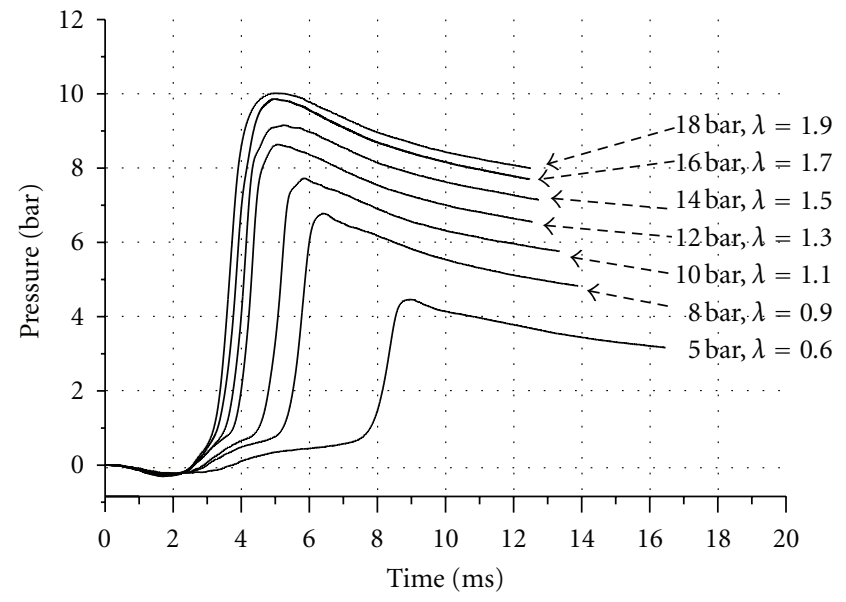

(a)

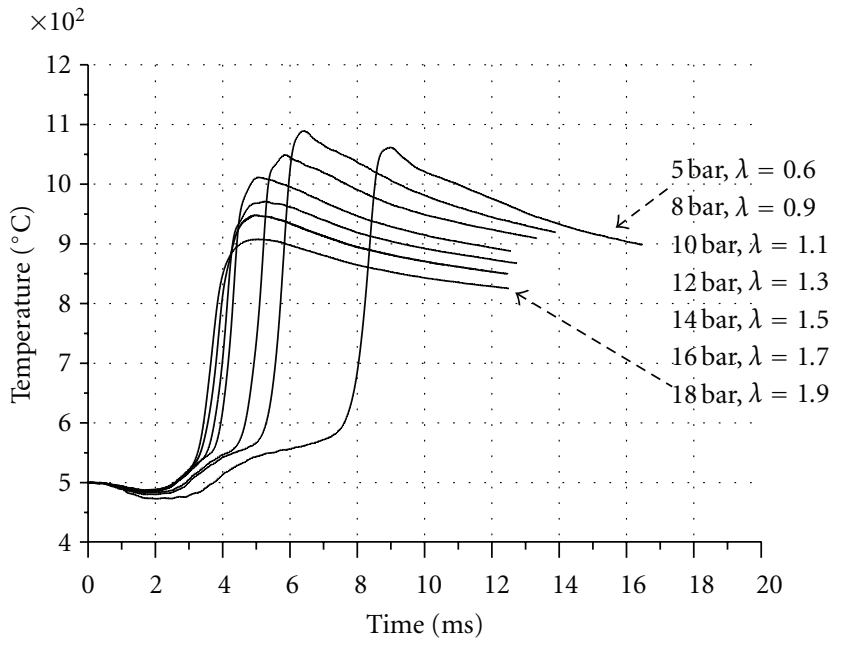

(b)

FIgURe 4: Pressure distribution (a) and temperature history (b) of the process in a free volume in time after Diesel injection starts at initial temperature $500^{\circ} \mathrm{C}$ and different initial pressures (mass of injected fuel is constant $23.8 \mathrm{mg}$ ).

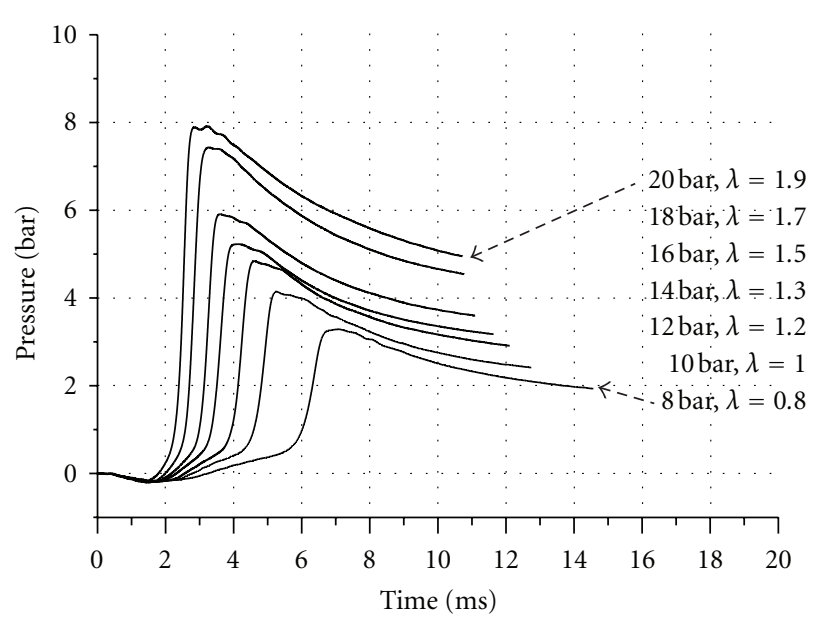

(a)

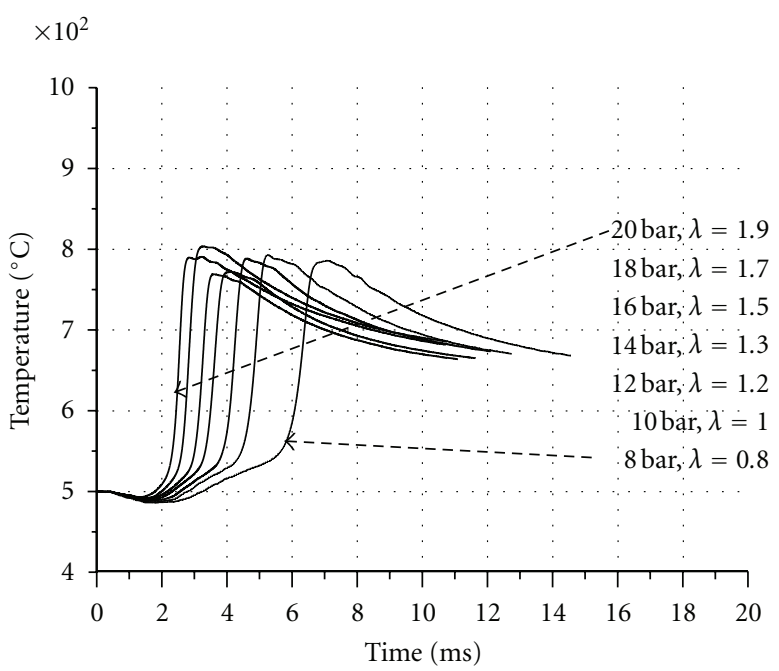

(b)

Figure 5: Pressure distribution (a) and temperature history (b) of the process in a SiC porous reactor having pore density 8 ppi in time after Diesel injection starts at initial reactor temperature $500^{\circ} \mathrm{C}$ and different initial pressures (mass of injected fuel is constant $23.8 \mathrm{mg}$ )

is performed for porous reactor (see Figure 5). The data are plotted at $T_{\mathrm{PM}-\mathrm{IB}}=500^{\circ} \mathrm{C}$ for different initial pressures $p_{\mathrm{A}-\mathrm{IB}(\mathrm{RR})}$. Pressure history shows reduced pressure peaks as compared to free volume system, and heat release process is qualitatively similar to free volume heat combustion. Reduced pressure peaks correspond to the heat accumulated in the porous reactor. Also, the corresponding combustion temperatures are reduced. The maximum temperature peaks in the porous reactor are much less dependent on air excess ratio than in a free volume system. This is due to the effect of heat accumulation in the reactor according to reactor heat capacity and heat transfer to the reactor solid phase. According to discussion made in Section 2, higher pore density and larger specific surface area of the porous reactor should result in more effective heat transfer to the reactor and increased heat accumulation in the solid phase of porous reactor. This effect can be observed in Figure 6 showing distribution of maximum combustion temperature after fuel injection in the case of free volume system and maximum gas temperature in porous reactors having different pore densities ( 8 ppi and $30 \mathrm{ppi}$ ). For reactor of higher pore density, the maximum temperature is significantly reduced at all investigated initial pressures. Almost constant temperature recorded in real porous reactors independently of initial pressure indicates the role of heat capacity and heat transfer conditions defining amount of heat accumulated in the porous reactors. This effect is observed for all investigated initial temperatures. 


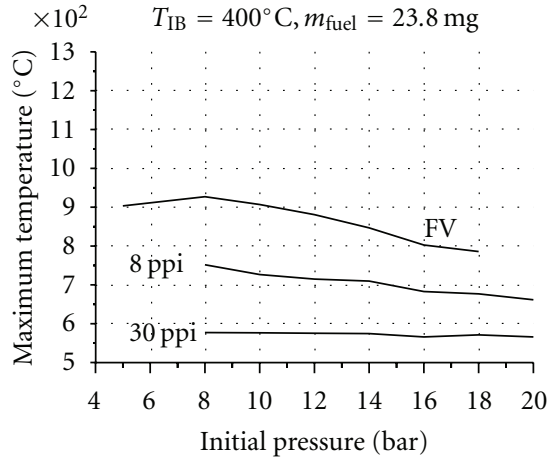

(a)

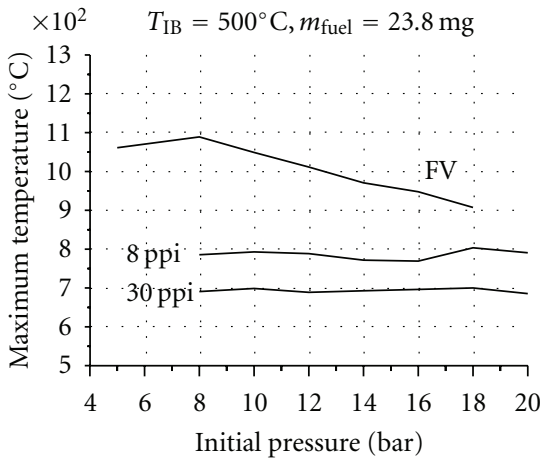

(b)

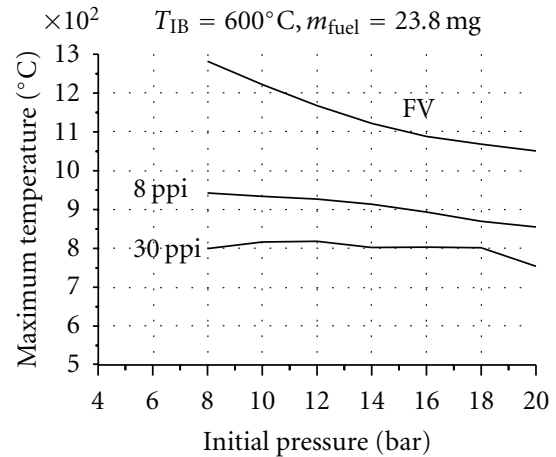

(c)

FIGURE 6: Distribution of maximum combustion temperature versus initial pressure in free volume system (FV) and in two porous reactors (8 ppi and $30 \mathrm{ppi}$ ) at three initial temperatures $400^{\circ} \mathrm{C}, 500^{\circ} \mathrm{C}$, and $600^{\circ} \mathrm{C}$ for a constant mass of injected fuel.

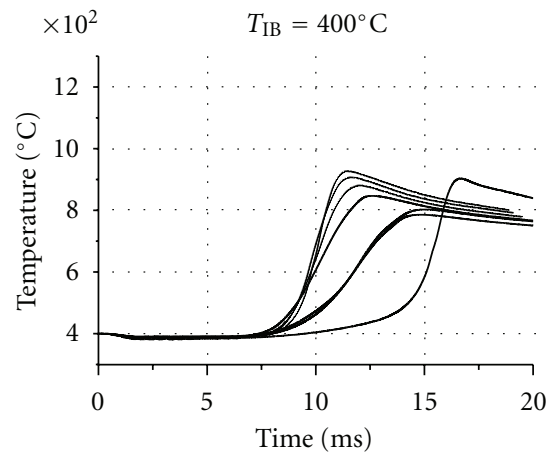

(a)

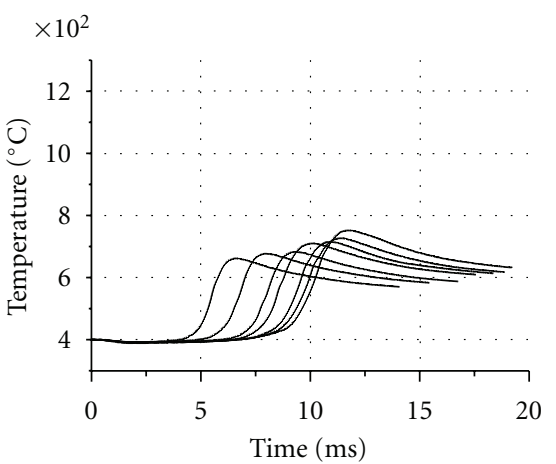

(d)

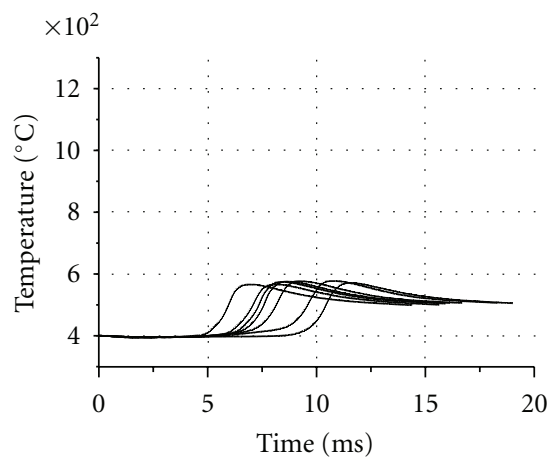

(g)

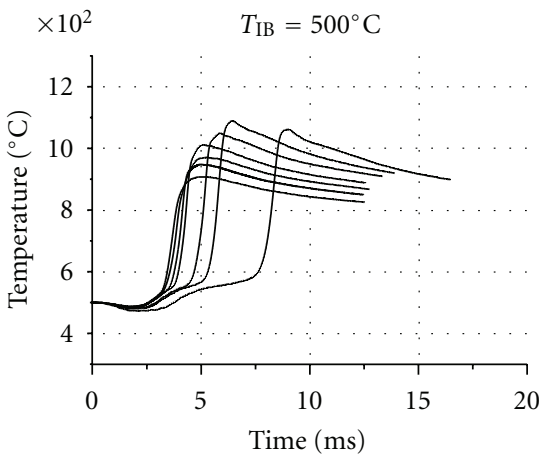

(b)

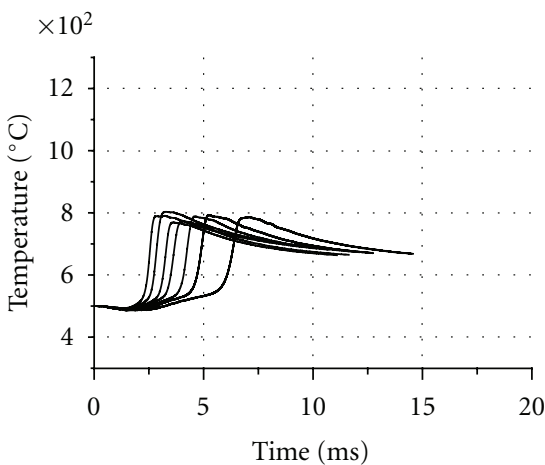

(e)

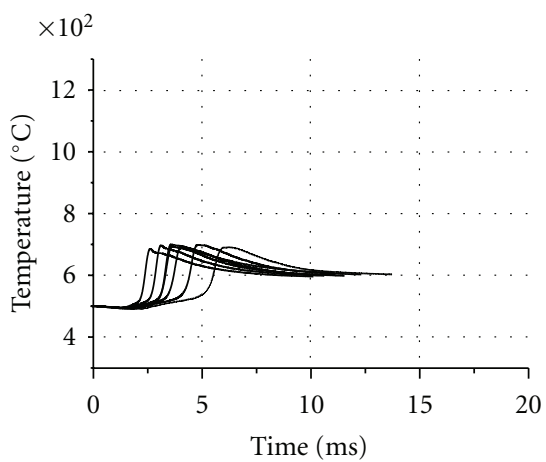

(h)

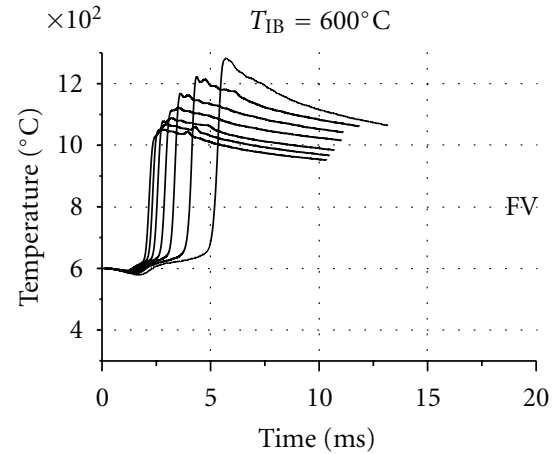

(c)

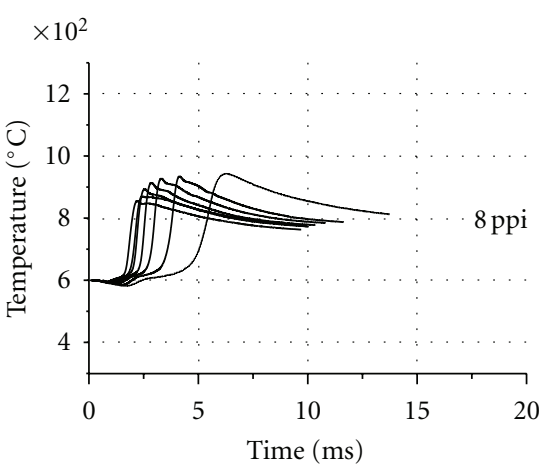

(f)

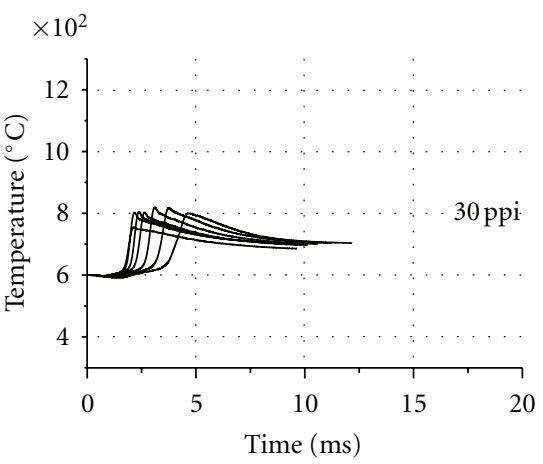

(i)

FIGURE 7: Temperature history after Diesel injection starts in free volume system (top), in low-pore-density SiC reactor 8 ppi (middle), and in high-pore-density $\mathrm{SiC}$ reactor $30 \mathrm{ppi}$ at three initial temperatures $400^{\circ} \mathrm{C}, 500^{\circ} \mathrm{C}$, and $600^{\circ} \mathrm{C}$ for different initial pressures from 8 to 20 bar (mass of injected fuel is constant $23.8 \mathrm{mg}$ ). 


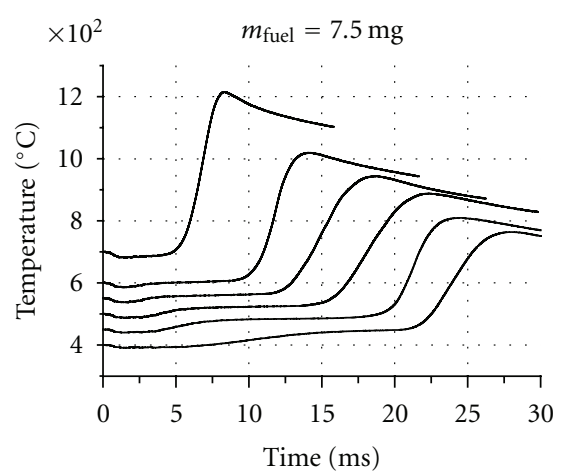

(a)

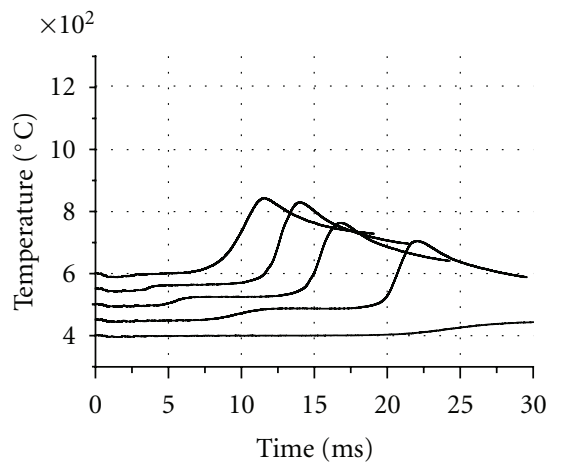

(d)

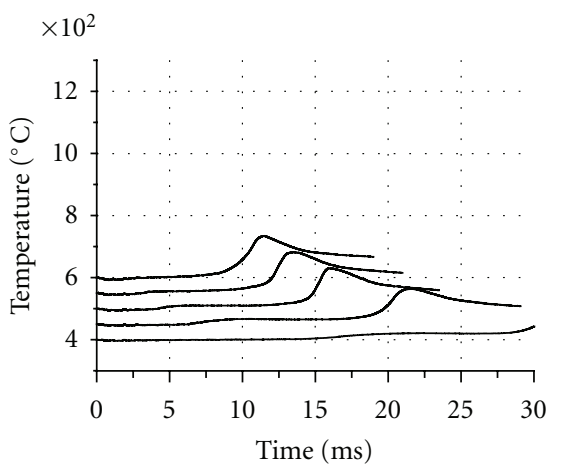

(g)

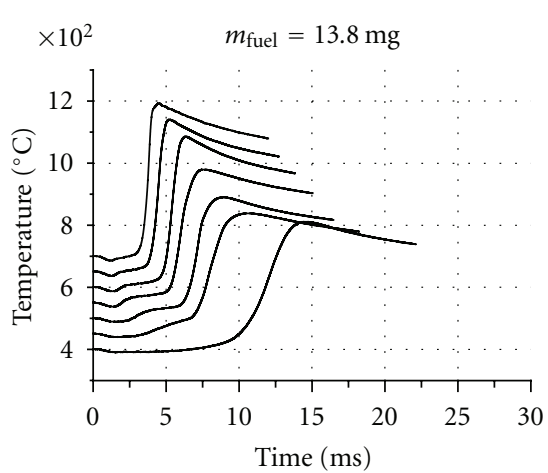

(b)

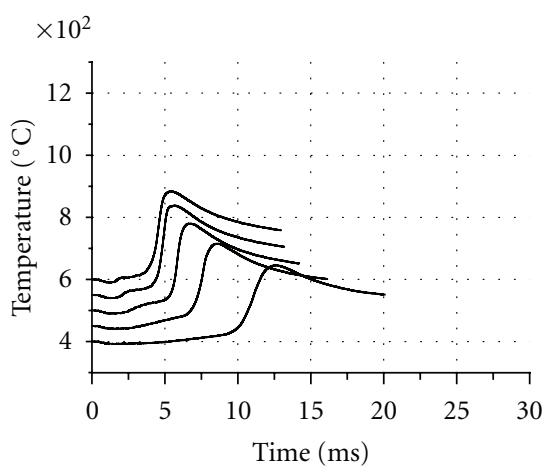

(e)

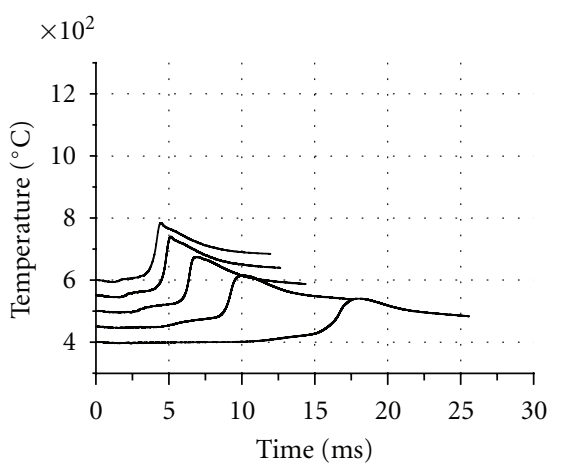

(h)

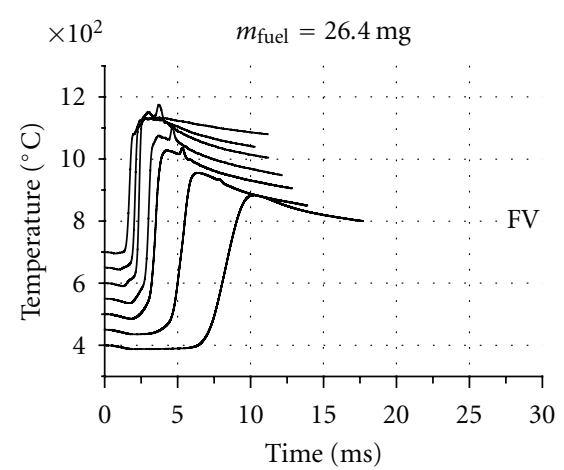

(c)

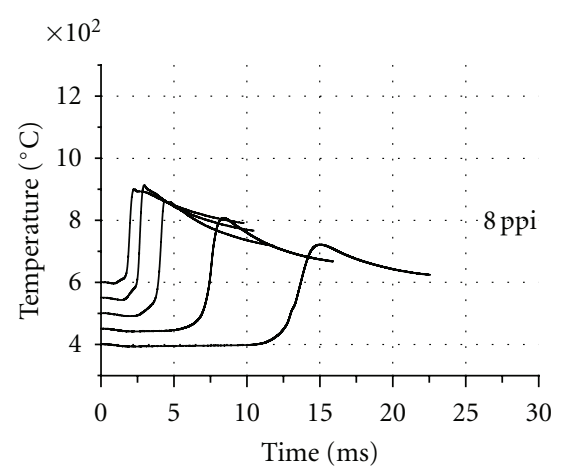

(f)

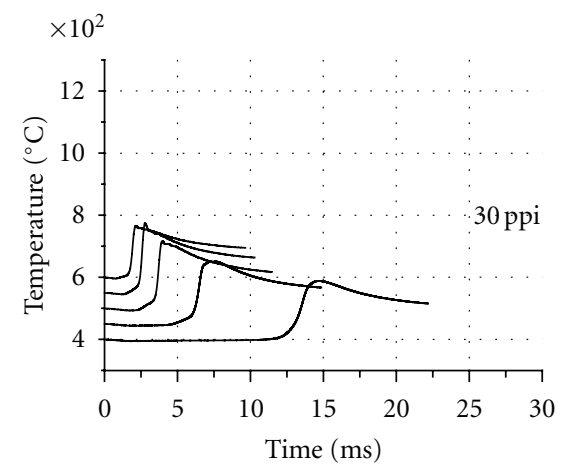

(i)

Figure 8: Temperature history after Diesel injection starts in free volume system (top), in low-pore-density SiC reactor 8 ppi (middle), and in high-pore-density $\mathrm{SiC}$ reactor $30 \mathrm{ppi}$ for three mass of injected fuel (7.5 mg, $13.8 \mathrm{mg}$, and $26.4 \mathrm{mg}$ ) for a constant air excess ratio (initial temperatures and initial pressures varies).

The effect of initial temperature on the combustion temperature histories in free volume as well as in two porous reactors is investigated in Figure 7. Individual curves correspond to particular initial chamber pressure and to a given air excess ratio. As already indicated for free volume combustion, the process is significantly dependent upon initial pressure and air excess ratio. An "irregular" dependence of the combustion temperature on the initial chamber pressure is observed at lowest presented temperature $\left(400^{\circ} \mathrm{C}\right)$. This "irregular" dependence means that the heat release process not necessarily gradually becomes faster with increasing pressure but is characterized by a range of pressures where the process is the fastest. Weclas et al. [16] have defined a positive pressure coefficient (PPC) corresponding to slightly lower temperatures and described nonregular dependence of reaction rate and delay time on the initial chamber pressure. Heat release process in porous reactors is faster and especially at lower initial temperature significantly accelerated as compared to free volume combustion (delay time). The combustion temperature (gas temperature according to the discussion in Section 2.2) in the real porous reactor is much less dependent on air excess ratio as a result of energy transport from the gas phase to the solid phase of the reactor. Significantly reduced temperature level is due to large heat capacity of the reactor and effective heat transfer (accumulation) in porous reactor. This gas temperature reduces with heat capacity and heat transfer (pore density and specific surface area) of the reactor. A nearly constant 


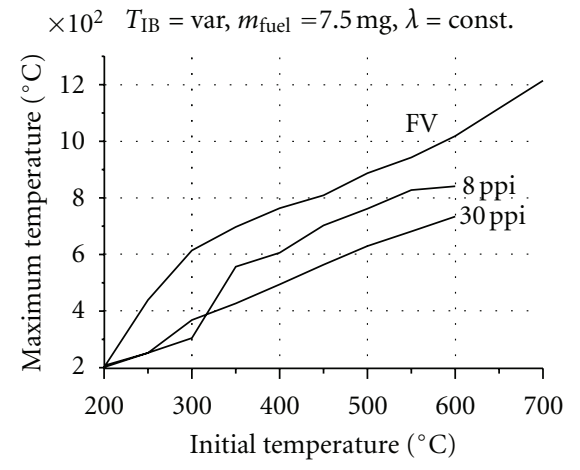

(a)

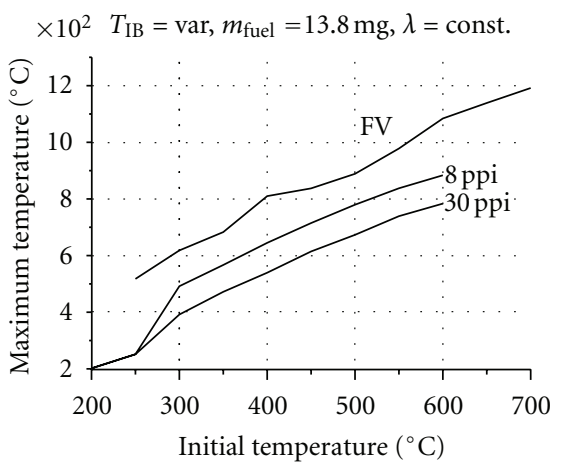

(b)

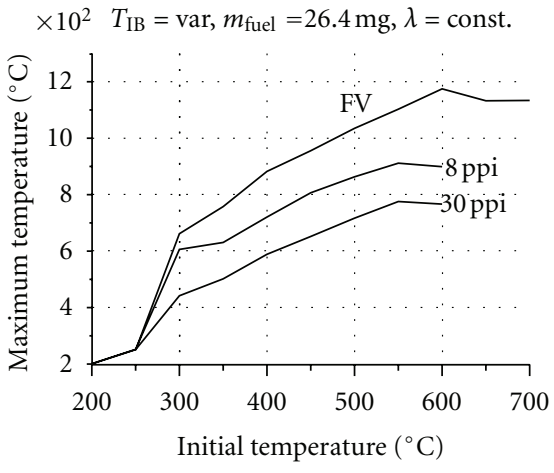

(c)

FIGURE 9: Distribution of maximum combustion temperature versus initial temperature in free volume system (FV) and in two porous reactors (8 ppi and $30 \mathrm{ppi}$ ) for three mass of injected fuel $7.5 \mathrm{mg}, 13.8 \mathrm{mg}$, and $26.4 \mathrm{mg}$ and a constant air excess ratio.

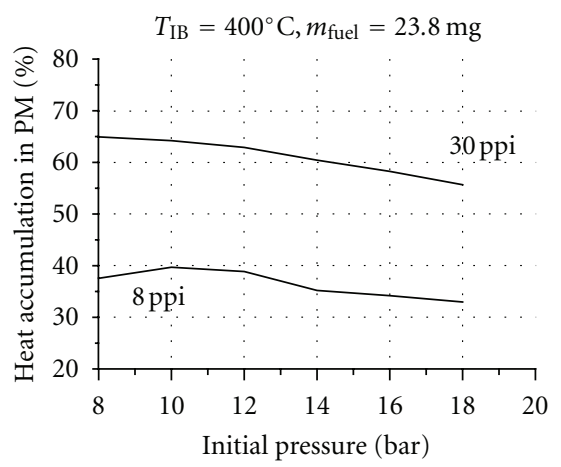

(a)

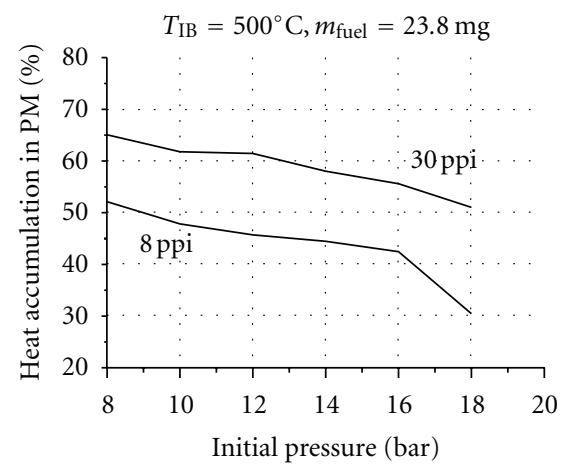

(b)

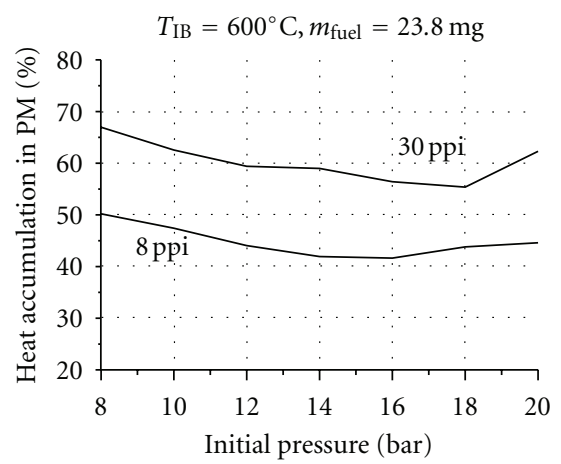

(c)

FIGURE 10: Distribution of amount of heat accumulated in porous reactors ( 8 ppi and $30 \mathrm{ppi}$ ) versus initial pressure at three initial reactor temperatures $400^{\circ} \mathrm{C}, 500^{\circ} \mathrm{C}$, and $600^{\circ} \mathrm{C}$ for a constant mass of injected fuel.

gas temperature in porous reactor at $600^{\circ} \mathrm{C}$ indicates the effect of limited amount of heat to be transferred to real reactor (contrary to assumption made for ideal reactor). A critical influence on heat accumulation in porous reactor and for resulting combustion temperature is the amount of supplied heat. This amount of supplied heat is directly controlled by the mass of fuel injected into the combustion system. Figure 8 shows comparison of the gas temperature for three different mass of fuel injected to the free volume combustion chamber or to porous reactor. Data are plotted for different initial temperatures at different initial pressures. Air excess ratio for a free volume system is 1.5 and for porous reactors is 1.35 . The process is analyzed in a limited period of time corresponding to initial $30 \mathrm{~ms}$ after fuel injection starts. The effect of amount energy supplied to the system together with heat accumulation in the reactor is clearly indicated. Combination of low amount of heat (low mass of injected fuel) with high pore density of the porous reactor results in only small change of the gas temperature as comparing to a free volume system. In the former case, most of energy is accumulated in a solid phase of the porous reactor significantly reducing the gas temperature change. The maximum temperature distribution is presented in Figure 9. Generally, the maximum combustion temperature gradually increases with increasing initial chamber temperature and is significantly reduced in porous reactors as compared to free volume combustion. This reduced temperature is a result of heat accumulation in a porous reactor, and the maximum temperature reduces with increasing reactor heat capacity, pore density, and specific surface area.

According to simplified analysis presented in Section 2.4, the corresponding amount of heat accumulated in porous reactor is shown in Figure 10. With increasing initial pressure, the heat release process is faster, and, under real reactor conditions, less heat can be transferred to the reactor. Generally, reactor having higher pore density can accumulate more heat under the same conditions. This analysis must, however, consider differences in fuel injection and mixture formation conditions inside the porous reactors having different pore densities. Such differences in process conditions may result in different efficiency of energy conversion into heat.

\section{Concluding Remarks}

A real porous reactor was considered under Diesel enginelike conditions simulated in a special combustion chamber. Thermodynamic conditions of the heat release process in combustion reactor have been analyzed. For a comparison, 
the same analysis was performed for a free volume (Diesel) combustion chamber. In all presented investigations, a real Diesel engine fuel injection and mixture formation conditions have been applied. Such a complex nonhomogeneous and time-dependent conditions are expected in a real engine with combustion in porous reactor. First of all, the effect of reactor heat capacity, pore density, specific surface area, and pore structure on the thermodynamic of the heat release process has been indicated. It was observed that heat accumulated in a solid phase of porous reactor significantly influences the thermodynamic conditions of the process. In an ideal reactor, it was assumed that the gas trapped inside reactor volume has the same temperature as the reactor temperature at any instant of time after fuel injection starts. Results obtained confirmed that it is important to consider real reactor conditions under which the gas and porous reactor temperatures are not the same. This effect is influenced by initial pressure and temperature (modeling conditions at TDC of compression) and by reactor parameters (heat capacity, pore density, specific surface area, pore structure). The temperature of the gas trapped in porous reactor volume during the heat release process is less dependent on air excess ratio as it is the case for free volume system. According to the reactor heat capacity and mixture formation conditions (fuel distribution, vaporization, and mixing in reactors having different pore densities), the maximum combustion temperature is significantly lowered as compared to free volume combustion chamber. This temperature, in all considered systems, is dependent on initial pressure. As expected from the analytical model of the porous reactor, the maximum pressure change during the heat release process in porous reactor is much less than in a free volume system. However, it must be underlined that the qualitative behavior of the heat release process in porous reactors and in free volume combustion chamber is similar. Also, the time scale of the process is similar. These similarities may support applicability of this kind of combustion to real engine conditions utilizing differences in thermodynamic conditions of the process.

\section{Acknowledgments}

M. Weclas thanks the German Ministry of Education and Research (BMBF) and Research Council (AiF) for financial support of the presented investigation (Project no. 17N2207). The authors thank Mr. Björn Leykauf and Mr. Iker Igartua García for their contribution to this investigation.

\section{References}

[1] V. S. Babkin, A. A. Korzhavin, and V. A. Bunev, "Propagation of premixed gaseous explosion flames in porous media," Combustion and Flame, vol. 87, no. 2, pp. 182-190, 1991.

[2] V. V. Martynenko, R. Echigo, and H. Yoshida, "Mathematical model of self-sustaining combustion in inert porous medium with phase change under complex heat transfer," International Journal of Heat and Mass Transfer, vol. 41, no. 1, pp. 117-126, 1998.
[3] A. A. M. Oliveira and M. Kaviany, "Nonequilibrium in the transport of heat and reactants in combustion in porous media," Progress in Energy and Combustion Science, vol. 27, no. 5, pp. 523-545, 2001.

[4] D. Trimis and F. Durst, "Combustion in a porous mediumadvances and applications," Combustion Science and Technology, vol. 121, no. 1-6, pp. 153-168, 1996.

[5] F. Durst, M. Keppler, and M. Weclas, "Air-assisted nozzle applied to very compact, ultra-low emission porous medium oil-burner," in the 3rd International Workshop on SPRAY, Lampoldshausen, Germany, 1997.

[6] M. A. Mujeebu, M. Z. Abdullah, A. A. Mohamad, and M. Z. A. Bakar, "Trends in modeling of porous media combustion," Progress in Energy and Combustion Science, vol. 36, no. 6, pp. 627-650, 2010.

[7] F. Durst and M. Weclas, "Porous Medium (PM) combustion technology and its application to internal combustion engines: a new concept for a near-zero emission engine," in Applied Optical Measurements, M. Lehner and D. Mewes, Eds., Springer, 1999.

[8] F. Durst and M. Weclas, "A new type of internal combustion engine based on the porous-medium combustion technique," Proceedings of the Institution of Mechanical Engineers, Part D, vol. 215, no. 1, pp. 63-81, 2001.

[9] F. Durst and M. Weclas, "A new concept of I.C. engine with homogeneous combustion in Porous Medium (PM)," in the 5th International Symposium on Diagnostics and Modeling of Combustion in Internal Combustion Engines (COMODIA '01), Nagoya, Japan, 2001.

[10] M. Kaviany, "In cylinder-thermal regeneration: Porous-Foam engine regenerator," in Principles of Heat Transfer in Porous Media, Springer, New York, NY, USA, 1999.

[11] C. W. Park and M. Kaviany, "Evaporation-combustion affected by in-cylinder, reciprocating porous regenerator," Journal of Heat Transfer, vol. 124, no. 1, pp. 184-194, 2002.

[12] M. Polasek and J. Macek, "Homogenization of Combustion in Cylinder of CI Engine Using Porous Medium,” SAE Technical Paper 2003-01-1 085, 2003.

[13] H. Liu, M. Xie, and D. Wu, "Simulation of a porous medium (PM) engine using a two-zone combustion model," Applied Thermal Engineering, vol. 29, no. 14-15, pp. 3189-3197, 2009.

[14] L. Schlier, W. Zhang, N. Travitzky, P. Greil, J. Cypris, and M. Weclas, "Macro-cellular silicon carbide reactors for nonstationary combustion under piston engine-like conditions," International Journal of Applied Ceramic Technology, vol. 8, no. 5, pp. 1237-1245, 2011.

[15] M. Weclas, "Potential of porous media combustion technology as applied to internal combustion engines," Journal of Thermodynamics, vol. 2010, Article ID 789262, 39 pages, 2010.

[16] M. Weclas, J. Cypris, and T. M. A. Maksoud, "Combustion of Diesel sprays under real-engine like conditions: analysis of low- and high-temperature oxidation processes, ILASS Europe 2010," in the 23rd Annual Conference on Liquid Atomization and Spray Systems, Brno, Czech Republic, September 2010, Paper no.ID ILASS10-40.

[17] M. Weclas, "Porous media in internal combustion engines," in Cellular Ceramics-Structure, Manufacturing, Properties and Applications, M. Scheffler and P. Colombo, Eds., Wiley, 2005.

[18] M. Weclas, "High velocity CR Diesel jet impingement on to porous structure and its utilization for mixture homogenization in I.C. engines," in the Drop/wall interaction: Industrial applications, Experiments and Modeling Workshop (DITICE '06), Bergamo, Italy, May 2006. 
[19] M. Weclas and R. Faltermeier, "Diesel jet impingement on small cylindrical obstacles for mixture homogenization by late injection strategy," International Journal of Engine Research, vol. 8, no. 5, pp. 399-413, 2007.

[20] M. Weclas, "Some fundamental observations on the diesel jet destruction and spatial distribution in highly porous structures," Journal of Porous Media, vol. 11, no. 2, pp. 125$144,2008$.

[21] M. Weclas, "Homogenization of liquid distribution in space by Diesel jet interaction with porous structures and small obstacles," in the 22nd European Conference on Liquid Atomization and Spray Systems, Como, Italy, September 2008, Paper no. ID ILASS08-A003.

[22] M. Weclas and J. Cypris, "'Distribution-nozzle" concept: a method for Diesel spray distribution in space for charge homogenization by late injection strategy, ILASS-Europe 2010," in the 23rd Annual Conference on Liquid Atomization and Spray Systems, Brno, Czech Republic, September 2010, Paper no. ID ILASS10-39.

[23] M. Weclas and J. Cypris, "Characterization of low- and hightemperature oxidation processes under non-premixed Dieselengine like conditions," submitted to International Journal of Engine Research. 

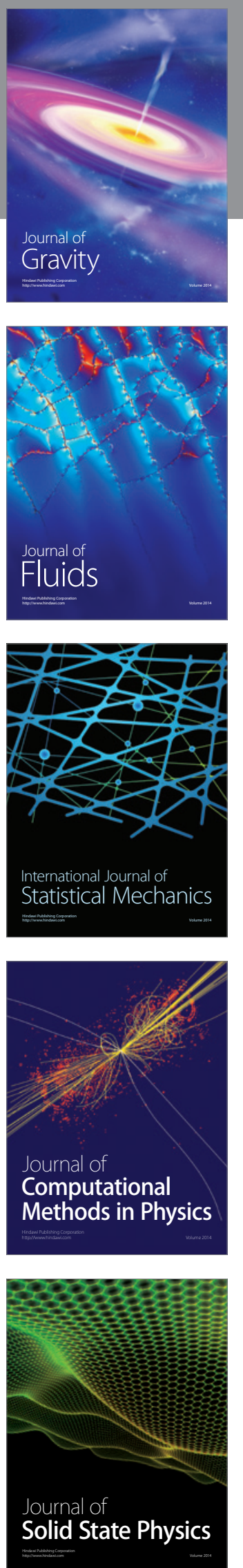

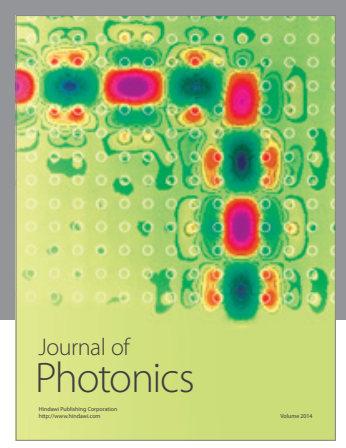

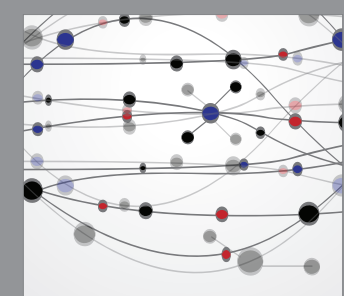

The Scientific World Journal
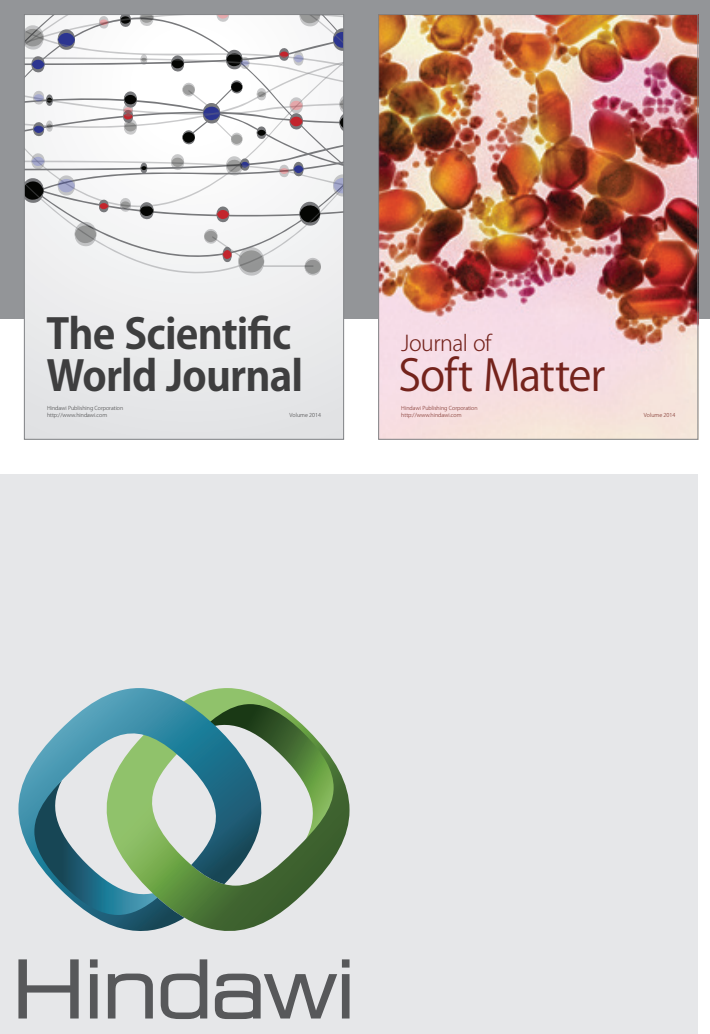

Submit your manuscripts at

http://www.hindawi.com
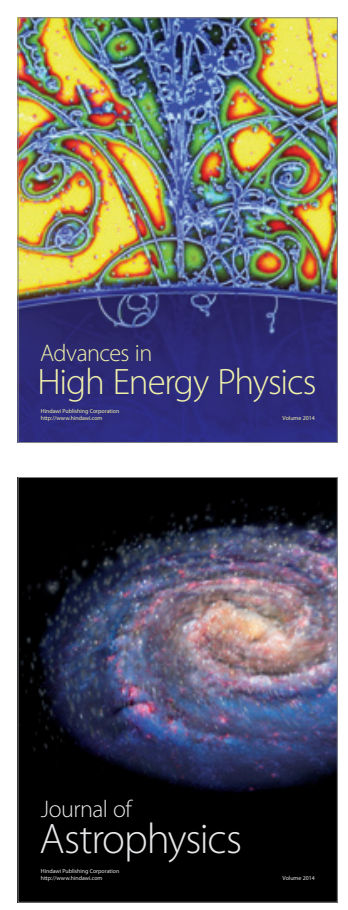
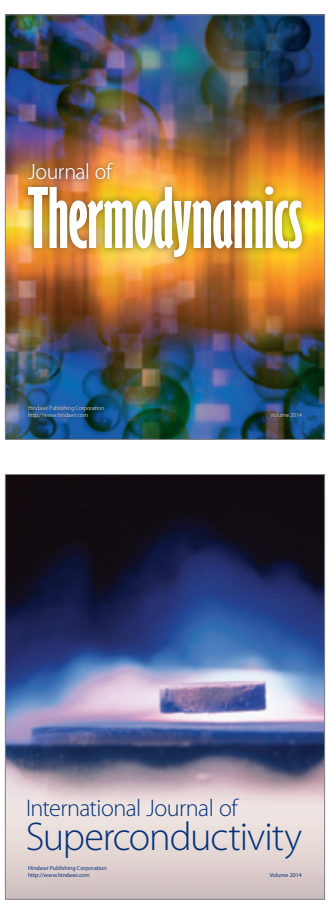
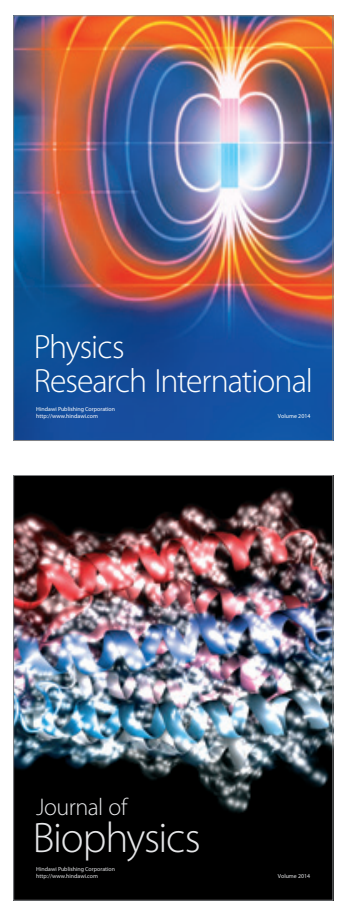
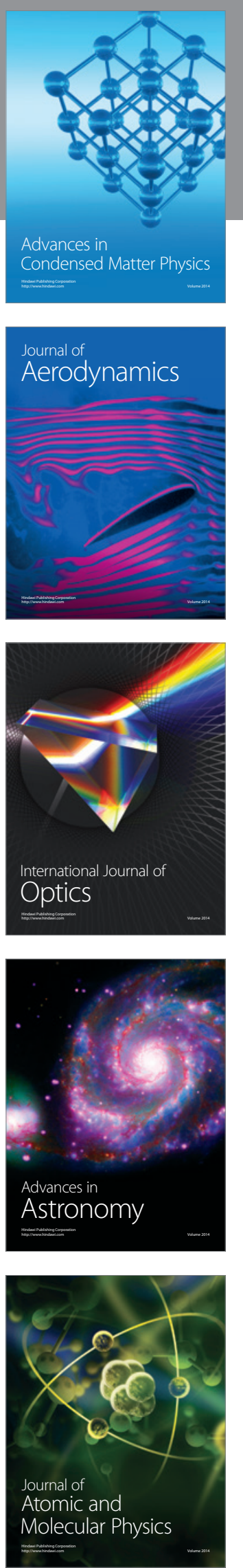